\title{
Andromeda and the seven dwarfs
}

\author{
Alan McConnachie $\dagger^{1}$, Mike Irwin ${ }^{1}$, Scott Chapman ${ }^{2}$, Rodrigo Ibata $^{3}$, \\ Annette Ferguson ${ }^{4}$, Geraint Lewis ${ }^{5}$, and Nial Tanvir ${ }^{6}$ \\ ${ }^{1}$ Institute of Astronomy, Madingley Road, Cambridge, CB3 0HA, U.K. \\ email: alan@ast.cam.ac.uk \\ 2 California Institute of Technology, Pasadena, CA, 91125 \\ ${ }^{3}$ Observatoire de Strasbourg, 11, rue de l'Universite, F-67000, Strasbourg, France \\ ${ }^{4}$ Institute for Astronomy, University of Edinburgh, Royal Observatory, Blackford Hill, \\ Edinburgh, EH9 3HJ, U.K. \\ ${ }^{5}$ Institute of Astronomy, School of Physics, A29, University of Sydney, NSW 2006, Australia \\ ${ }^{6}$ Physical Sciences, Univ. of Hertfordshire, Hatfield, AL10 9AB, U.K.
}

\begin{abstract}
The satellite system of M31 is the next closest satellite system to our own, and is well within reach of current generations of ground-based instrumentation. We have conducted a survey of this system with the Isaac Newton Telescope Wide Field Camera, particularly focussing on the seven dwarf spheroidal companions, and derived homogeneous, fundamental information for them. This reveals the presence of severe tidal distortion and multiple structural components in some systems. In addition, a comparison of the M31 dwarf spheroidals to their Galactic counterparts shows that the M31 dwarf spheroidals have scale radii generally twice as large as the Galactic dwarfs, suggesting that the tidal field of M31 may be substantially weaker than that for the Galaxy. A Keck/DEIMOS study of the newly discovered satellite And IX suggests that this galaxy has an anomalously large metallicity for its luminosity, and shows it to be strongly dark matter dominated.
\end{abstract}

Keywords. (galaxies:) Local Group, galaxies: dwarf, galaxies: stellar content, galaxies: structure, galaxies: kinematics and dynamics

\section{Introduction}

Most of our detailed knowledge on the structure of dwarf spheroidal (dSph) galaxies comes from observations of the nine dSphs which make up part of the Galactic satellite system - Draco, Ursa Minor, Carina, Fornax, Sculptor, Sextans, Sagittarius, Leo I and Leo II. For these, it is possible to reach below the main sequence turn-off with modest instrumentation and conduct a detailed analysis of their stellar populations. Irwin, \& Hatzidimitriou (1995) were the first to analyse the structure of these systems by mapping their resolved star counts, providing much fundamental structural information. Generally, they found that the stellar structure of all the dSphs was well described by a single component King model or an exponential profile. A generic feature was an excess of stars at large radii, beyond the best-fitting tidal radius. These stars have generally been interpreted as a sign of tidal disturbance from interaction with the gravitational potential of the Milky Way, although other interpretations are possible. Choi, Guhathakurta \& Johnston (2002) have shown that a break in the radial profile - which may lead to an excess of stars observed in this way - is a natural consequence of tidal perturbation.

$\dagger$ New address from October 2005: Department of Physics and Astronomy, University of Victoria, 3800 Finnerty Road, Victoria, BC V8P 1A1, Canada 
More recent studies have shown that the structure of dSphs may be more complex than that which has previously been assumed. Harbeck, et al. (2001) conducted a systematic and homogeneous study of population gradients in several Local Group dSphs, by looking for spatial variations in the colour of the red giant branch and the horizontal branch. They find evidence for gradients in many of the systems analysed. Tolstoy, et al. (2004) have since analysed the global population of stars in Sculptor from photometry and kinematic information and showed that it has a spatially, chemically and kinematically distinct second component, in the form of a more centrally concentrated core. Additionally, Kleyna, et al. (2004) have shown that the Sextans dSph has a kinematically cold core. They postulate that this is a disintegrating star cluster, whose orbit has decayed by dynamical friction to the centre of Sextans. This is similar to Ursa Minor, which shows a distinct concentration of stars offset from the geometric center of this galaxy (Irwin, \& Hatzidimitriou (1995), Kleyna, et al. (2003)) and also shows evidence of kinematic substructure at the location of the secondary over-density.

Given these findings in the Galactic dSph system, it is timely to look closely at the next closest dSph system to our own, that of M31. Approximately 16 satellites make up this subsystem, of which seven - Andromeda I, II, III, V, VI, VII, and IX - are classified as dSphs. In comparison to the dSphs which orbit the Galaxy, relatively little is known in detail about the stellar populations of the Andromeda dSphs. Deep HST fields have been obtained for Andromeda I, II and III which extend below the horizontal branch (Da Costa et al. (1996), Da Costa et al. (2000), Da Costa et al. (2002)). Horizontal branch analysis shows that each has had an extended epoch of star formation and variations in their horizontal branch morphology suggest that their star formation histories have been notably different. In addition, Andromeda I is observed to display a gradient in its horizontal branch morphology, such that their are more blue horizontal branch stars located at larger radius from the center of the dwarf (Da Costa et al. (1996)). Some evidence for AGB components have also been seen in these systems (most recently by Kerschbaum, et al. (2004) and Harbeck, et al. (2004)), although a strong intermediate age component similar to some of the Galactic dSphs is generally lacking.

We have obtained Johnston V and Gunn i photometry for the majority of the members of the M31 subgroup using the Isaac Newton Telescope Wide Field Camera (INT WFC). Figure 1 shows two typical dSph colour magnitude diagrams (CMDs) created from our data for And I \& II. This photometry is deep enough to observe the top few magnitudes of the red giant branch in each system. We have used this to conduct a homogeneous study of these satellites and derive fundamental information for them, including distances, metallicities, and structural parameters for the dSphs. Detailed descriptions of these studies are to be found in McConnachie, et al. (2004), McConnachie, et al. (2005), McConnachie \& Irwin (2005a), McConnachie \& Irwin (2005b). The main M31 survey is detailed in Ferguson, et al. (2002). In addition, we have conducted a spectroscopic analysis of the newest dSph, And IX, using the Keck/DEIMOS spectrograph (Chapman, et al. (2005)). Here, we present a broad overview of this study and some of the main results.

\section{Distances and metallicities}

The tip of the red giant branch (TRGB) is an ideal standard candle for older, more metal poor systems. Physically, this stage in stellar evolution represents the point of the core helium (He) flash of low mass stars. During this phase of stellar evolution the temperature of the degenerate, quasi-isothermal He core is only dependent upon the properties of the thin hydrogen burning shell around it. This, in turn, varies only very slightly with chemical abundance and surrounding mass. The bolometric luminosity of 


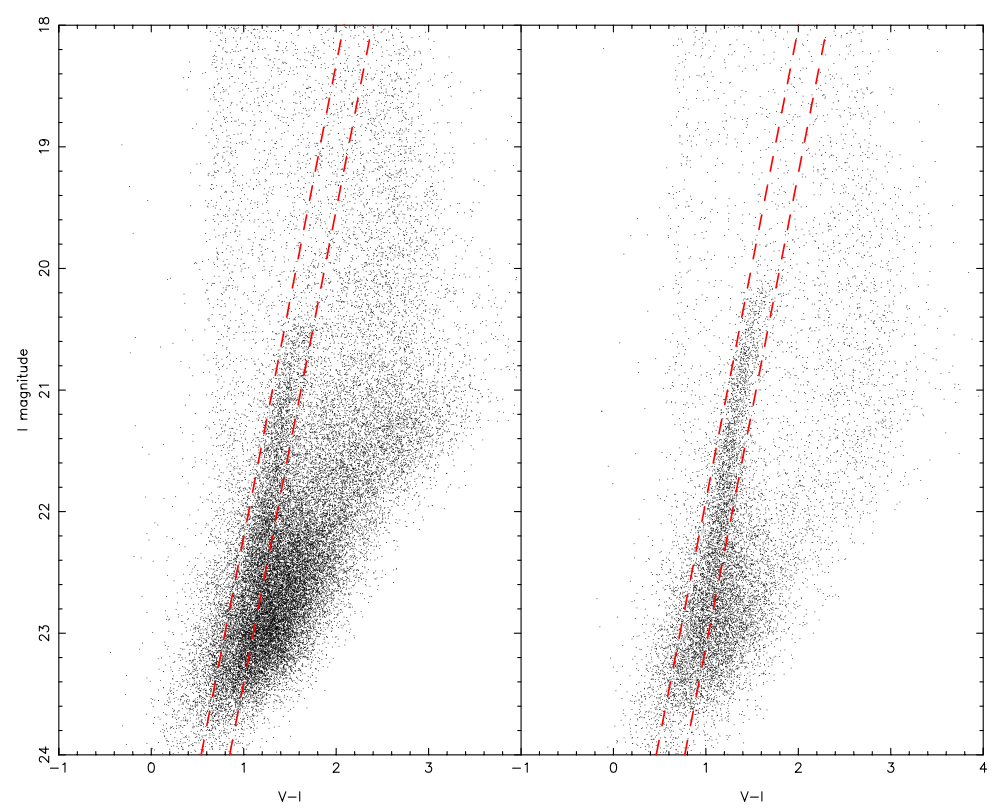

Figure 1. CMDs for And I (left panel) and And II (right panel) demonstrating the quality of the INT WFC photometry. The red giant branch of each system is clearly visible. The additional red feature in the And I CMD is due to the M31 giant stellar stream discovered as part of our main M31 survey.

the TRGB is thus of roughly constant intrinsic brightness. The absolute I band magnitude of the TRGB $\left(M_{I}^{T R G B}\right)$ is also found to be nearly constant for these stars as the stellar spectrum peaks near this wavelength range and absorption effects in the stellar atmosphere are minimal. For more metal rich stars, molecular absorption causes $M_{I}^{T} R G B$ to dim. Barker, Sarajedini \& Harris (2004) have recently conducted a study of the reliability of the TRGB as a distance indicator for stellar populations with a wide variety of star formation histories and find that it is reliable for any system which is not dominated by a metal-rich population $([\mathrm{Fe} / \mathrm{H}]>-0.3)$ or a population with a substantial young $(\lesssim 1.7$ Gyrs) component. This makes it ideal for determining distances to Local Group galaxies.

The TRGB is clearly visible in Figure 1 for both And I \& II. In McConnachie, et al. (2004), we devise an algorithm in order to measure this point, based upon a generalised least-squares fitting procedure. This allows the distances to be derived for each of the 17 galaxies for which we have INT WFC data. In order to account for the slight metallicity dependency of $M_{I}^{T R G B}$, we also self-consistently derive the median metallicity of each system based upon the colour of its red giant branch. The net result is a large, homogeneous set of distances and metallicities for the Local Group galaxies M31, M33, And I, II, III, V, VI, VII, IX, NGC 205, 185, 147, Pegasus, WLM, LGS3 and Aquarius. The results are listed in Table 1 and 2 of McConnachie, et al. (2005), and are ideal for studies of Local Group galaxy properties. As an example, Figure 2 is a projection of the M31 satellite system in M31-centric spherical coordinates, derived from these estimates. The positions of these satellites around M31 are much better constrained than previously, as systematic errors have been minimised and each distance is accurate to $\sim 5 \%$. 


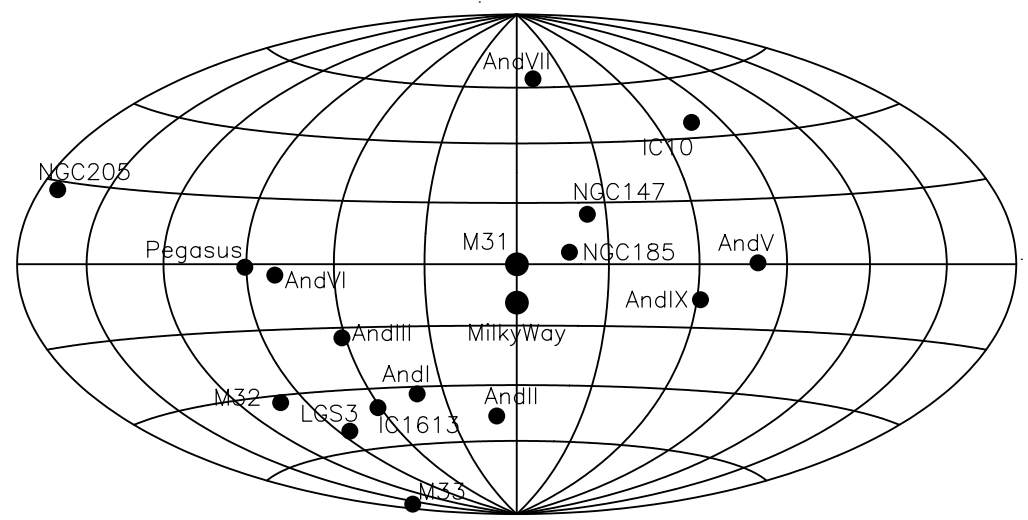

Figure 2. An Aitoff projection of the satellite galaxies of M31 in a native coordinate system. $l$ is measured around the disk of M31 (where the Milky Way is defined to lie at $l=0$ ) and $b$ is measured from the disk of M31. The position of each satellite around M31 is better constrained than previous studies due to the homogeneous set of distances used.
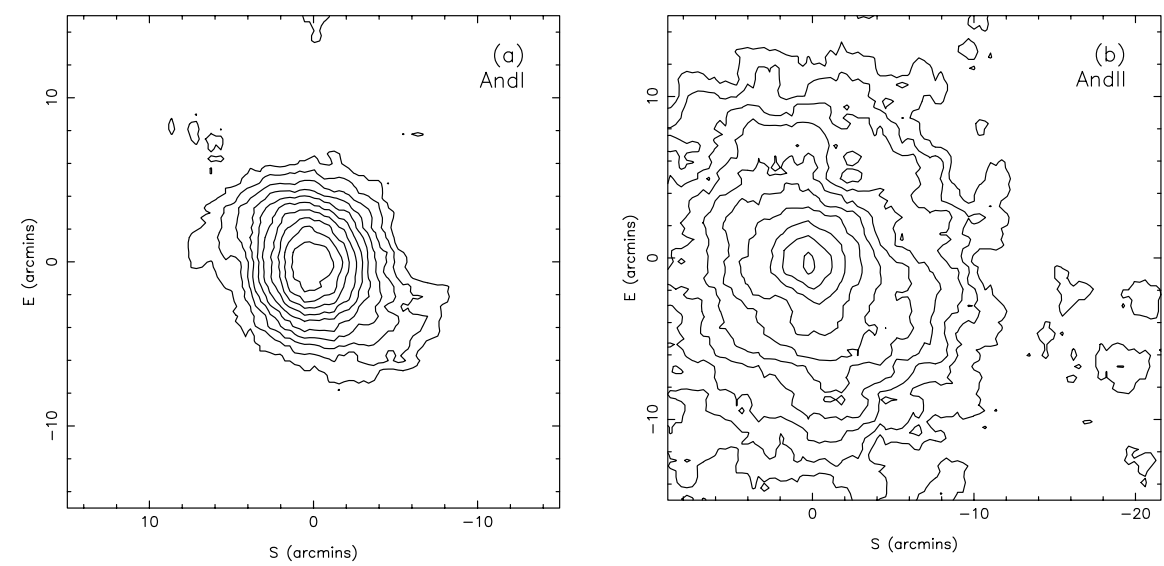

Figure 3. Isopleth maps for the And I \& II (left and right panels respectively) based upon resolved star counts. The outermost contours are $2 \sigma$ above the background. And I shows clear evidence of severe tidal disruption, whereas And II is tidally unperturbed. This galaxy, however, shows compelling evidence for a secondary 'core' component (Figure 4).

\section{Structural properties of the M31 dSphs}

Figure 3 shows contour maps of the red giant branch spatial distribution for And I (left panel) and And II (right panel). The outer contour is $2 \sigma$ above the background in both plots. Both of these galaxies are relatively large, and have distinct morphologies.

And I is a very non-spheroidal dSph, and displays a distinct and very well-defined S-shape. This is an unambiguous indicator of strong tidal disruption, and it is possible that And I will have a low surface brightness tidal stream associated with it. In addition, And I may have a relatively low density dark matter halo surrounding it, as this would otherwise act to shield the stellar component from severe tidal disruption. Several other M31 dSphs also show tentative evidence for tidal disturbance, but none as convincing as And I.

And II, on the other hand, is nearly perfectly circular in projection, and displays no evidence of tidal effects. However, examination of its isopleths shows that the central few contours appear more concentrated that the outer contours. Figure 4 shows the surface 


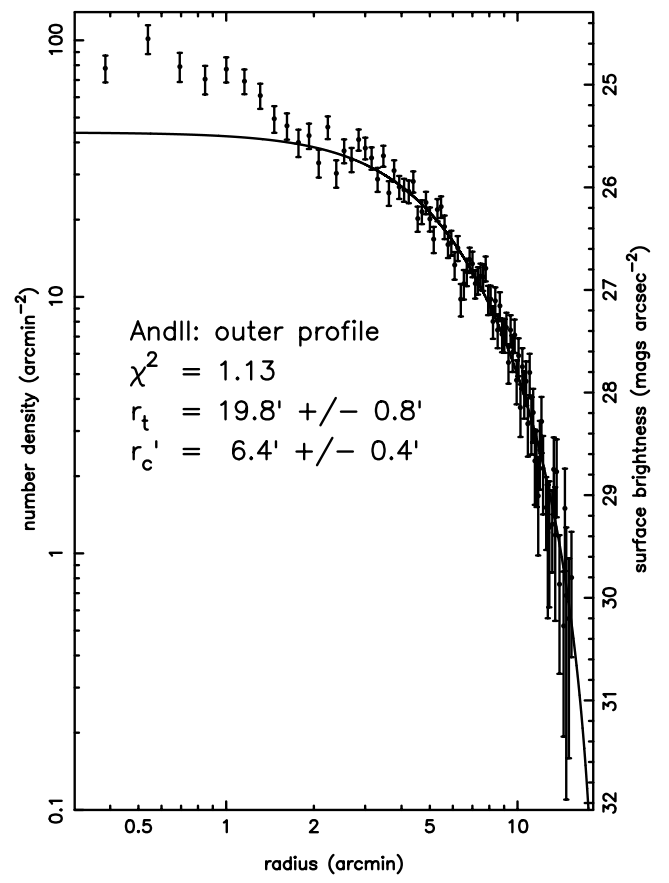

Figure 4. The best fit King profile to the surface brightness profile of Andromeda II, ignoring the inner $2^{\prime}$. The formal value of the fit is very good, and the divergence of the King profile to the data at small radii suggests the presence of a secondary core component in this dSph.

brightness profile of And II, derived from the stars counts. The solid line is a King profile fit to the outer regions of the profile, and clearly demonstrates that there is a break in the profile and a large excess of stars at small radii. This 'core' is reminiscent of the sort of structure seen by Tolstoy, et al. (2004), and provides compelling evidence that And II is also a two-component dSph. Deeper global photometry extending below the horizontal branch is required.

\section{The Local Group dSph populations compared}

The central surface brightnesses, integrated magnitudes, half light radii and scale radii of the best fitting King, exponential and Plummer profiles are derived for And I, II, III, V, VI, VII and Cetus in McConnachie \& Irwin (2005a). Coupled with the distance information to each of these systems, this allows us to compare the structural characteristics of the M31 dSph population to their Galactic counterparts.

As an example of the sort of comparison that is possible, Figure 5 shows the half-light radii (left panel) and tidal radii of the best-fitting King profile (right panel) as a function of integrated luminosity for the M31 dSphs (triangles), Galactic dSphs (squares) and the isolated Cetus dSph (diamond). For a given absolute magnitude, the M31 dSphs are generally at least twice as extended as the Galactic dSphs. This is true both in terms of the half-light radius and also in terms of the tidal radii. It may be that this reflects environmental differences. For example, if the tidal field of M31 is significantly less than for the galaxy, then we would expect the M31 population of dSphs to have generally larger tidal radii. It is not so clear, however, how tidal effects could significantly affect the half-light radii to produce the trend that we observe. 

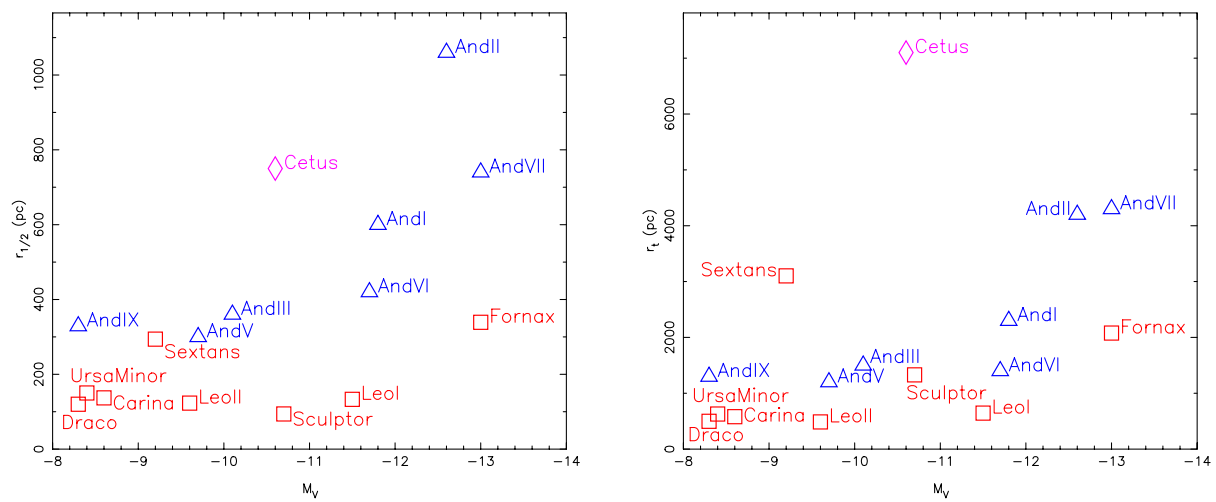

Figure 5. Half-light radii (left panel) and tidal radii (right panel) for the Local Group dSphs. Satellites of M31 are shown as triangles, satellites of the Galaxy are shown as squares, and the isolated dSph in Cetus is shown as a diamond. For a given absolute magnitude, the scale radii of the M31 dSphs are generally at least twice as large as their Galactic counterparts. This could reflect environmental differences between the populations.

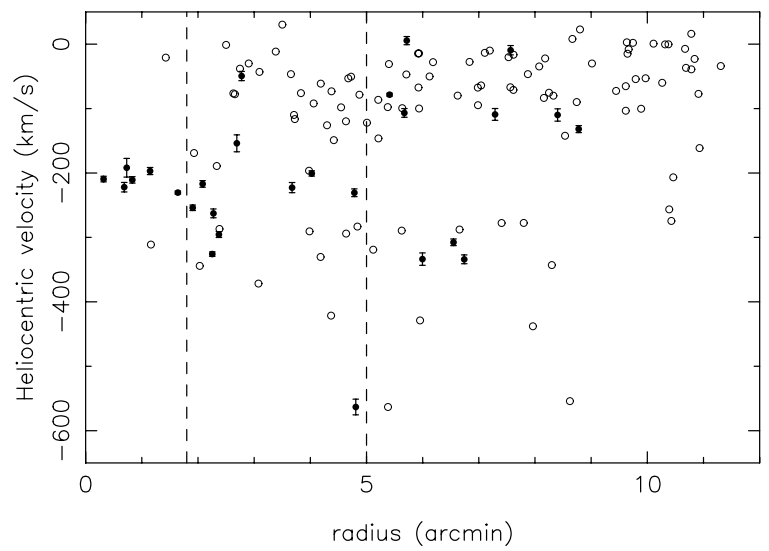

Figure 6. Radial velocities of all the star's in our Keck/DEIMOS field centred on And IX. Black points represent stars which lie on the And IX red giant branch. The vertical dashed lines are 1.8 and 5 arcmins from the center of M31. Due to the close proximity of And IX to M31, there is significant contamination in this field. However, within 1.8 arcmins of the center there is a clear kinematic grouping of stars with the correct colour to belong to And IX. We identify these as And IX red giant branch stars.

\section{Kinematics of the faintest dSph, Andromeda IX}

The discovery of And IX has only recently been reported by Zucker, et al. (2004) and a detailed study of its stellar content is not yet available, although it appears as if And IX consists only of an old stellar population, with no evidence of young or intermediateage components. As part of our group's ongoing kinematic survey of M31 using the DEep Imaging Multi-Object Spectrograph on the Keck II telescope, we have conducted a kinematic study of this object to constrain its dynamical evolution and dark matter content (Chapman, et al. (2005).

Figure 6 shows the measured radial velocities of all the stars in our Keck/DEIMOS field centered on And IX. The black points represent stars which additionally have colours and magnitudes which place them on the red giant branch of And IX in a CMD. And IX is sufficiently close to M31 that there is significant contamination from M31 in our DEIMOS field. However, by applying a tight spatial cut then we can identify a group of stars within 

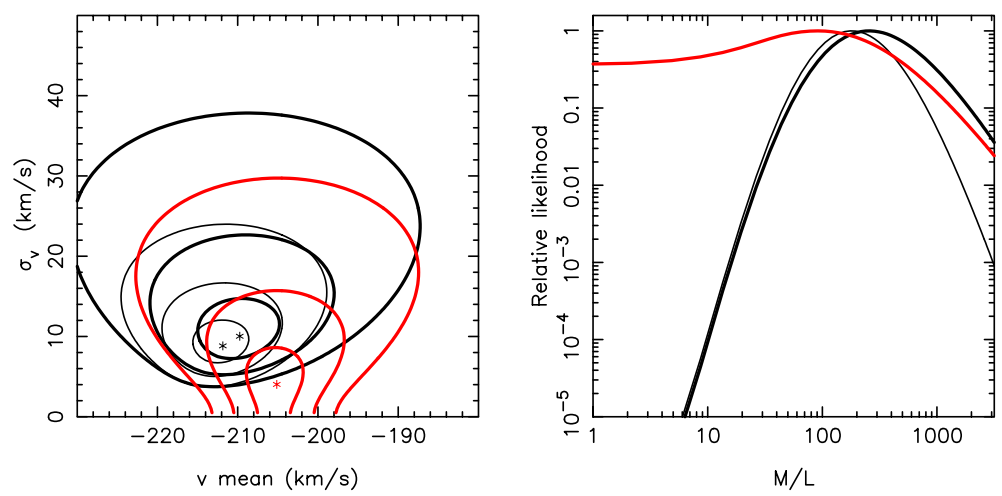

Figure 7. Left panel: Maximum likelyhood contours for the radial velocity and velocity dispersion of And IX. The dark line corresponds to the analysis using all six stars within 1.8 arcmins highlighted in Figure 6 . The lighter line is the same analysis but neglecting the star whose velocity is furthest from the mean of this group. Right panel: Relative probability for the mass-to-light ratio of And IX. Dark and light lines are the same as in the left panel.

1.8 arcmins of the center of And IX which are tightly correlated in radial velocity and which have the correct colours to be And IX red giant branch stars. We identify these six stars as And IX members and conduct a maximum likelyhood analysis of these stars to obtain the radial velocity and dispersion of And IX. The corresponding maximum likelyhood contours are shown as the darkest lines in the left panel of Figure 7 and imply a radial velocity for And IX of $v_{r}=210 \pm 5 \mathrm{~km} \mathrm{~s}^{-1}$, with a velocity dispersion of $\sigma=10_{-3}^{+5} \mathrm{~km} \mathrm{~s}^{-1}$. The darkest line in the right panel of Figure 7 is the relative probability for the mass-to-light ratio assuming And IX is not very tidally disturbed. We find that the mass-to-light ratio of And IX is $>27$ at the $99 \%$ confidence level.

Of the six stars on which our analysis is performed, it is very unlikely that any of them belong instead to M31. If, however, the star whose velocity is furthest from the mean in our grouping is actually from M31, then our results change dramatically - the velocity dispersion of And IX would then be consistent with zero within the $1 \sigma$ errors. This is represented by the lighter lines in Figure 7. Nevertheless, this is unlikely.

By stacking the six spectra, we are able to measure the equivalent width of the Calcium triplet and imply a metallicity. This corresponds to $[\mathrm{Fe} / \mathrm{H}]=-1.5$ dex. This is approximately 0.5 dex higher than would be expected from the faint luminosity of And IX. It may be that the chemical enrichment processes in this faintest of galaxies differs from many other dSph galaxies in the Local Group.

\section{Summary}

Our INT WFC photometric survey of M31's satellites has allowed the derivation of a large number of fundamental parameters for this subsystem, including distances and metallicities, and reveals the presence of tidal effects and multiple components in several of the dSphs. In addition, important difference appear to exist in the scale sizes of the M31 and Galactic dSphs, which may reflect environmental differences. Our Keck/DEIMOS survey of And IX suggest that And IX is a highly dark matter dominated satellite, and that the metallicity is anomalously large for such a faint system, perhaps suggesting that And IX is an atypical dSph.

\section{Acknowledgements}

AM would like to thank the organisers for a very enjoyable and exciting meeting, and the IAU for providing financial assistance. 


\section{References}

Barker, M.K., Sarajedini, A. \& Harris, J. 2004 ApJ 606, 869

Bellazzini, M., Ferraro, F.R. \& Pancino, E. 2001 ApJ 556, 635

Chapman, S., Ibata, R., Lewis, G., Ferguson, A., Irwin, M. \& McConnachie, A.W. \& Tanvir, N. 2005 ApJ submitted

Da Costa, G.S. \& Armandroff, T.E. 1990 AJ 100, 162

Da Costa, G.S., Armandroff, T.E., Caldwell, N. \& Seitzer, P. 1996 AJ 112, 2576

Da Costa, G.S., Armandroff, T.E. \& Caldwell, N. 2000 AJ 119, 705

Da Costa, G.S., Armandroff, T.E. \& Caldwell, N. 2002 AJ 124, 332

Choi, P.I., Guhathakurta, P. \& Johnston, K.V. 2002 AJ 124, 310

Ferguson, A., Irwin, M.J., Ibata, R.A., Lewis, G.F. \& Tanvir, N. 2002 AJ 124, 1452

Harbeck, D., Gallagher, J.S. \& Grebel, E.K. 2004 AJ 127, 2711

Harbeck, D., Grebel, E.K., Holtzman, J., Guhathakurta, P., Brandner, W., Geisler, D., Sarajedini, A., Dolphin, A., Hurley-Keller, D. \& Mateo, M. 2001 AJ 122, 3092

Irwin, M. \& Hatzidimitriou, D. 1995 MNRAS 277, 1354

Kerschbaum, F., Nowotny, W., Olofsson, H. \& Schwarz, H.E. 2004 A $\& A$ 427, 613

Kleyna, J.T., Wilkinson, M.I., Evans, N.W. \& Gilmore, G. 2004 MNRAS 354, 543

Kleyna, J.T., Wilkinson, M.I., Gilmore, G. \& Evans, N.W. 2003 ApJL 588, L21

McConnachie, A.W., Irwin, M.J., Ferguson, A., Ibata, R.A., Lewis, G.F. \& Tanvir, N. 2004 MNRAS 350, 243

McConnachie, A.W. \& Irwin, M.J. $2005 M N R A S$ submitted

McConnachie, A.W. \& Irwin, M.J. 2005 MNRAS submitted

McConnachie, A.W., Irwin, M.J., Ferguson, A., Ibata, R.A., Lewis, G.F. \& Tanvir, N. 2005 MNRAS 356, 979

Tolstoy, E., Irwin, M.J., Helmi, A., Battaglia, G., Jablonka, P., Hill, V., Venn, K.A., Shetrone, M.D., Letarte, B., Cole, A.A., Primas, F., Francois, P., Arimoto, N., Sadakane, K., Kaufer, A., Szeifert, T. \& Abel, T. 2005 ApJL 617, L119

Zucker, D.B, et al. 2004 ApJL 612, L121

\section{Discussion}

CALDwell: The excess light in the center of And III is intriguing, but I worry that the presence of foreground stars may have made your measurements more difficult there. Does your finding hold true even in areas not contaminated by these stars?

MCCONNACHIE: Yes, although foreground stars do complicate the analysis, it is possible to account for them in the analysis procedure and so there presence does not affect our conclusions.

BAILIN: How robust are the And IX results to the exclusion of any of the 6 stars from the spectrum?

MCCONNACHIE: If we exclude the most discrepant star of the 6 then the results change dramatically - the velocity dispersion is consistent with zero. However, it is very improbable that this star is an interloper.

MATEO: How far is And IX from the center of M31 in angular and linear units?

MCConnACHIE: Andromeda IX is approximately 1.5 to 2 degrees from the center of M31, and has a 3D separation from M31 of about $40 \mathrm{kpc}$. 